\title{
Short communication: Variability in fermentation end-products and methanogen communities in different rumen sites of dairy cows
}

\author{
ZhiYuan Ma, ${ }^{*} \dagger$ Rong Wang, ${ }^{*}$ Min Wang, ${ }^{* 1}$ XiuMin Zhang, ${ }^{*} \dagger$ HongXiang Mao, ${ }^{*}$ and ZhiLiang Tan* \\ ${ }^{*}$ Key Laboratory of Agro-Ecological Processes in Subtropical Region, South-Central Experimental Station of Animal Nutrition and Feed Science, \\ Ministry of Agriculture, Institute of Subtropical Agriculture, The Chinese Academy of Sciences, Changsha, Hunan 410125, \\ People's Republic of China \\ †University of Chinese Academy of Sciences, Beijing 100049, People's Republic of China
}

\section{ABSTRACT}

The objective of this study was to investigate differences in fermentation and methanogen communities in samples collected from 3 sites in the rumen of dairy cows. The study involved 3 ruminally cannulated nonlactating Chinese Holstein dairy cows fed a diet of $40 \%$ forage and $60 \%$ concentrate feeds. Four handfuls of whole ruminal contents were collected from the cranial sac, middle of the ventral sac, and caudodorsal blind sac of the rumen of the cows at $0,2.5$, and 6 $\mathrm{h}$ after the morning feeding. Concentrations of VFA, ammonia, and dissolved methane and hydrogen were analyzed. Methanogen populations and communities were analyzed targeting 16s rRNA genes. Dissolved methane concentration and $\mathrm{pH}$ were highest in samples from the cranial rumen. Ruminal fluid from the cranial rumen also had greater copy numbers of the Methanobrevibacter and higher Simpson indexes compared with samples from middle of the ventral rumen. In summary, cranial rumen had higher dissolved $\mathrm{CH}_{4}$ concentration than middle and hind rumen, which might be caused by the greater population of Methanobrevibacter with higher ruminal $\mathrm{pH}$.

Key words: rumen fermentation, methanogen, methane, hydrogen

\section{Short Communication}

Methane in the rumen is produced by a specialized group of microbes called methanogenic archaea, a process that prevents accumulation of hydrogen in the rumen. It was reported that dissolved $(\mathbf{d}) \mathrm{CH}_{4}$ was higher in ruminal fluid from the front, cranial sac of the rumen than from the middle rumen (Wang et al., 2016a), suggesting that $\mathrm{CH}_{4}$ production may vary within rumen sites. The cranial sac of rumen usually has a higher

Received November 6, 2017.

Accepted January 21, 2018.

${ }^{1}$ Corresponding author: mwang@isa.ac.cn
$\mathrm{pH}$ and lower VFA concentration compared with other rumen sites (Bryant, 1964; Duffield et al., 2004; Wang et al., 2016a), which may be caused by rumination and consequent entry of saliva (Bryant, 1964; Duffield et al., 2004; Wang et al., 2016a). Ruminal pH is an important factor influencing rumen methanogens (Lana et al., 1998; Wenner et al., 2017), and thus may affect methanogenesis and $\mathrm{dCH}_{4}$ distribution within the rumen. Studies are lacking concerning investigating the differences in methanogenesis and methanogen population among rumen sites.

This experiment was approved by the Animal Care Committee, Institute of Subtropical Agriculture, Chinese Academy of Sciences, Changsha, China. Three nonlactating Chinese Holstein rumen-fistulated (100mm internal diameter; Anscitech Co. Ltd., Wuhan, China) dairy cows $(\mathrm{BW}=460 \pm 36.5 \mathrm{~kg}, \mathrm{DMI}=6.25$ $\pm 0.690 \mathrm{~kg} / \mathrm{d}$, methane emission $=210 \pm 24.8 \mathrm{~L} / \mathrm{d}$; mean $\pm \mathrm{SD}$ ) were used in this experiment. The cows were housed in a tiestall barn, fed a TMR twice daily at 0600 and $1600 \mathrm{~h}$ to allow approximately $5 \%$ refusals, and had free access to fresh water. The TMR contained (DM basis) $40.0 \%$ corn silage, $24.0 \%$ corn grain, $6.0 \%$ soybean meal, $10.2 \%$ wheat middling, $13.2 \%$ dried distillers corn grain with solubles, $2.0 \%$ soybean oil, $0.5 \%$ sodium chloride, $1.7 \%$ calcium carbonate, $0.6 \%$ calcium hydrophosphate, $0.8 \%$ magnesium sulfate, and $1.0 \%$ vitamin/trace-mineral premix (Jiuding Feed Co. Ltd, Changsha, China).

Whole ruminal contents samples were collected by same person from 3 sites in the rumen, as described by McCracken et al. (1999). The sites were cranial sac, middle of the ventral sac, and caudodorsal blind sac of the rumen and were defined as front, middle, and hind rumen, respectively. Four handfuls of whole ruminal contents were collected from the 3 sites at $0,2.5$, and 6 $\mathrm{h}$ after the morning feeding, and sampling was repeated in 2 consecutive days. Ruminal fluid was separated by squeezing through 4 layers of cheesecloth.

The apparatus used to extract $\mathrm{dCH}_{4}$ and $\mathrm{dH}_{2}$ in the liquid fraction was as described in Wang et al. (2014). 
Extracted $\mathrm{CH}_{4}$ and $\mathrm{H}_{2}$ were analyzed using GLC (Agilent 7890A, Agilent Inc., Palo Alto, CA). Concentrations of $\mathrm{dH}_{2}$ and $\mathrm{dCH}_{4}$ in the original liquid fraction were calculated as described in Wang et al. (2016a).

The $\mathrm{pH}$ of the ruminal fluid was measured immediately after samples were collected using a portable pH meter (Starter 300; Ohaus Instruments Co. Ltd., Shanghai, China), which was calibrated before each sampling using standard buffers ( $\mathrm{pH} 4.0$ and 7.0). Concentrations of VFA were analyzed using GLC (Agilent 7890A, Agilent Inc., Palo Alto, CA) according to Wang et al. (2014). Concentration of ammonia was analyzed using the procedure of Weatherburn (1967).

Aliquots of ruminal fluid collected at $0,2.5$, and 6 $\mathrm{h}$ after the morning feeding were frozen immediately in liquid nitrogen and stored at $-80^{\circ} \mathrm{C}$. Two hundred fifty microliters of the ruminal fluid were pipetted into 2-mL enzyme-free centrifuge tubes for DNA extraction. Microbial DNA extraction of samples followed the protocol described by $\mathrm{Yu}$ and Morrison (2004). Three hundred microliters of TE buffer (Tris $10 \mathrm{mM}$, EDTA $1 \mathrm{~m} M, \mathrm{pH}=8.0$ ) was used to elute total DNA. The concentration and purity of total DNA were measured using a ND-2000 spectrophotometer (NanoDrop Technologies, Wilmington, DE). Quantitative real-time PCR (qPCR) was performed using the procedures detailed by Jiao et al. (2014). The qPCR assays were performed on a LightCycler 480 (Roche Molecular Systems Inc., Pleasanton, CA), with a total volume of $10 \mu \mathrm{L}$, using SYBR Premix Ex Taq (Takara Inc., Dalian, China). The PCR reaction contained 1 (template DNA) +5 (SYBER Green Mix, Takara Inc.) + 0.25 (forward primer) +0.25 (reverse primer) +3.5 (double-distilled $\left.\mathrm{H}_{2} \mathrm{O}\right)=10 \mu \mathrm{L}$. All samples were assayed in triplicate and all standard curves met the following requirements $\left(\mathrm{R}^{2}>0.99,90 \%<E<120 \%\right)$. The PCR efficiency, for sort $E$, is calculated by the slope of the standard curve $\left(E=10^{(- \text {slope)-1 }}\right)$. Details are described by Rutledge and Côté (2003). Forward (F) and reverse primer (R) selected from the literature for qPCR of methanogen groups were 5'-GGATTAGATACCCSGGTAGT-3'(F) and $\quad 5^{\prime}$-GTTGARTCCAATTAAACCGCA-3'(R) for total methanogens (Hook et al., 2010); 5'-CGWAGGGAAGCTGTTAAGT-3' (F) and 5'-TACCGTCGTCCACTCCTT-3' (R) for Methanobacteriales (Yu et al., 2005); and 5'-CCTCCGCAATGTGAGAAATCGC-3' (F) and 5'-TCWCCAGCAATTCCCACAGTT-3' (R) for Methanobrevibacter (Huang et al., 2016).

The template DNA representing 3 rumen sites for each cows used in PCR for sequencing of amplicons were pooled at equal volume ratio of total DNA extracted from ruminal fluid sampled from 3 time points on 2 consecutive days. The $16 \mathrm{~S}$ rDNA V4-V5 hyper- variable regions of the methanogen genomic DNA were used for PCR amplification with the primers 5'-TGYCAGCCGCCGCGGTAA-3' (524F) and 5'-YCCGGCGTTGAVTCCAATT-3' (Arch958R). The PCR reactions were performed in a triplicate $20-\mu \mathrm{L}$ mixture containing $0.8 \mu \mathrm{L}$ of each primer, $10 \mathrm{ng}$ of template DNA, $2 \mu \mathrm{L} 2.5 \mathrm{~m} M$ deoxyribonucleoside triphosphate, $0.4 \mu \mathrm{L}$ of FastPfu polymerase (Transgen, Beijing, China), and $4 \mu \mathrm{L} 5 \times$ FastPfu Buffer (Transgen). The thermal cycling programing was performed as 3 min for an initial denaturation step at $95^{\circ} \mathrm{C}, 27$ cycles of denaturation at $95^{\circ} \mathrm{C}$ for $30 \mathrm{~s}$, annealing at $55^{\circ} \mathrm{C}$ for $30 \mathrm{~s}$, and elongation at $72^{\circ} \mathrm{C}$ for $45 \mathrm{~s}$, with a final extension at $72^{\circ} \mathrm{C}$ for $10 \mathrm{~min}$. The PCR products were excised from $2 \%$ agarose gels and purified using a QIAquick Gel extraction kit (Qiagen, Hilden, Germany). Amplicons from each reaction mixture were quantified fluorometrically, normalized, and pooled at equimolar ratios based on the concentration of each amplicon. Amplicons were sequenced with the Illumina MiSeq platform (Illumina, San Diego, CA) at Majorbio Bio-Pham Technology (Shanghai, China). Raw reads were submitted to the NCBI Sequence Read Archive (SRA) database (https:// www.ncbi.nlm.nih.gov/sra) under accession number SRP121400. Quality control of the sequence reads were performed using MOTHUR v.1.39.5 (Schloss et al., 2009) and followed the protocol described by Kozich et al., (2013). The high-quality reads were clustered into operational taxonomic units (OTU) at 97\% similarity using Usearch v.7.0 (Edgar, 2013). Representative sequences defined by abundance from each OTU were using PyNAST (Caporaso et al., 2010) against SILVA archaea database v.128 (Quast et al., 2013). Taxonomy analysis were using the RDP classifier v.11.1 (Wang et al., 2007) with a minimum support threshold of $80 \%$. A principal coordinate analysis (PCoA) was performed based on Bray-Curtis similarity distances (Bray and Curtis, 1957).

Fermentation and $\mathrm{qPCR}$ data were averaged to get the mean value of 2 consecutive days, which were further analyzed statistically using the linear mixed model of SPSS 17.0 software (SPSS Inc., Chicago, IL), with rumen site $(\mathrm{n}=3)$, sampling time $(\mathrm{n}=3)$, and cow $(\mathrm{n}$ $=3)$ as fixed effects, sampling time $(\mathrm{n}=3)$ as repeated measures, and interaction between rumen site and cow. No interactions between rumen site and sampling time were found; thus, it was removed from the model. Diversity indexes estimated from 16s rRNA gene library sequences were performed using a linear mixed model with rumen site $(\mathrm{n}=3)$ and cow $(\mathrm{n}=3)$ as fixed effect. Significance was declared at $P \leq 0.05$.

Three cows were employed, and samples collected at 3 sampling times had great variabilities in $\mathrm{dCH}_{4}, \mathrm{dH}_{2}$, and molar percentage of acetate and propionate $(P$ 
Table 1. Effect of rumen site and sampling time on fermentation end-products in ruminal fluid of dairy cows $(\mathrm{n}=3)$

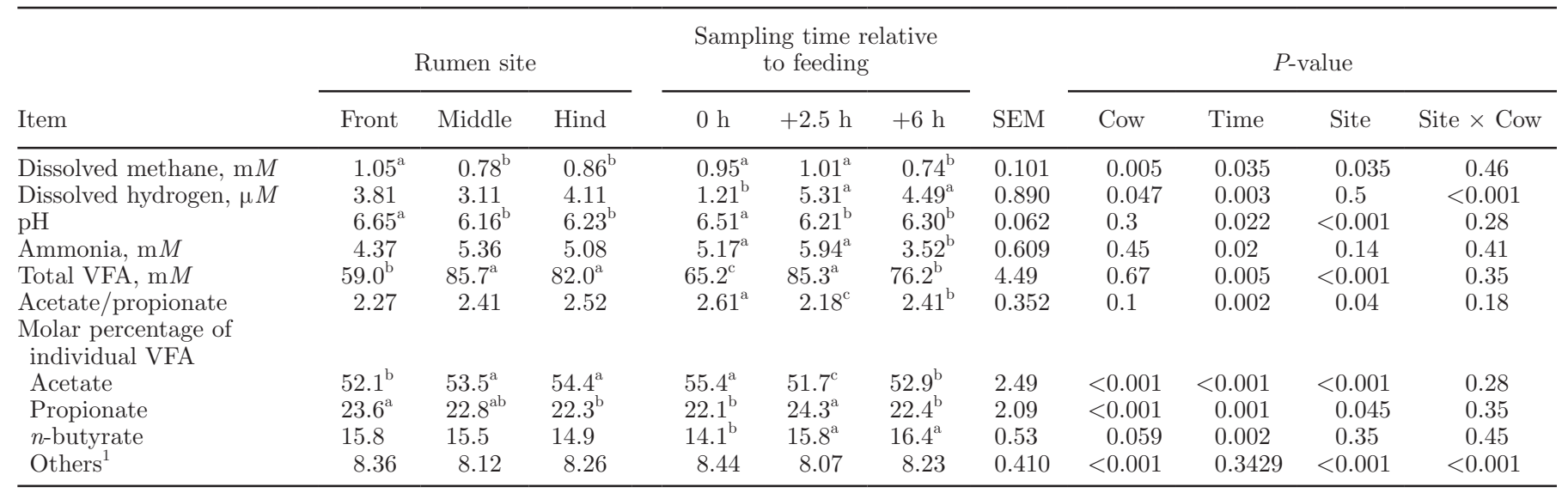

${ }^{a-c}$ Within rumen site or sampling time, means having different superscripts differ at $P \leq 0.05$.

${ }^{1}$ Including isobutyrate, isovalerate, and valerate.

$<0.05)$. Concentrations of $\mathrm{dCH}_{4}$ in samples from the front rumen were 34.6 and $22.1 \%$ greater $(P=0.035)$ than in samples from the middle and hind rumen, respectively (Table 1), which confirms results from our previous study (Wang et al., 2016a). The front rumen had the highest ruminal $\mathrm{pH}(P<0.001)$ and lowest VFA concentration $(P<0.001)$ among the 3 rumen sites. The cranial rumen sac is separated from the rest of the rumen by the cranial pillar and it is likely that large amounts of saliva flowing into the front rumen increase ruminal $\mathrm{pH}$ and dilute VFA (Li et al., 2009; Shen et al., 2012; Wang et al., 2016a). Even with the dilution effect of saliva, $\mathrm{dCH}_{4}$ concentration in samples from the front rumen was higher $(P=0.035)$, which suggests that methanogenesis in front rumen is more intensive than in other rumen sites. Rumen methanogenesis was also reported to be pH-dependent (Lana et al., 1998; Wenner et al., 2017); thus, higher $\mathrm{pH}$ in the front rumen may contribute to increased methanogenesis compared with the other 2 rumen sites in dairy cows.

Although the front rumen had the lowest acetate molar percentage $(P<0.001)$ and the highest $(P=0.045)$ propionate molar percentage among the 3 rumen sites, the molar concentration of propionate in the front rumen was 28.7 and $24.0 \%$ lower than in middle and hind rumen, respectively. The lower molar concentration of propionate and higher $\mathrm{dCH}_{4}$ in the front of rumen compared with other rumen sites can be expected, as methane and propionate typically are inversely related to one another, as both are electron sinks for reducing equivalents (Janssen, 2010; Wang et al., 2016a,b). However, other factors should be also considered to explain different VFA profile, such as the dilution effect of incoming saliva from reticulum and esophagus in the front of rumen ( $\mathrm{Li}$ et al., 2009) and variations in the efficiency of rumen mixing and host absorption of the individual VFA in 3 rumen sites (Aschenbach et al., 2011).

A total number of 328,913 valid sequences from 9 samples were analyzed and 123 OTU at $3 \%$ distance were obtained. A total of 7 orders were detected via taxonomic analyses against SILVA archaea database v.128. The most abundant orders were Methanobacteriales and Thermoplasmatales, which account 76.1 and $20.3 \%$, respectively, of total good reads. Thermoplasmatales is

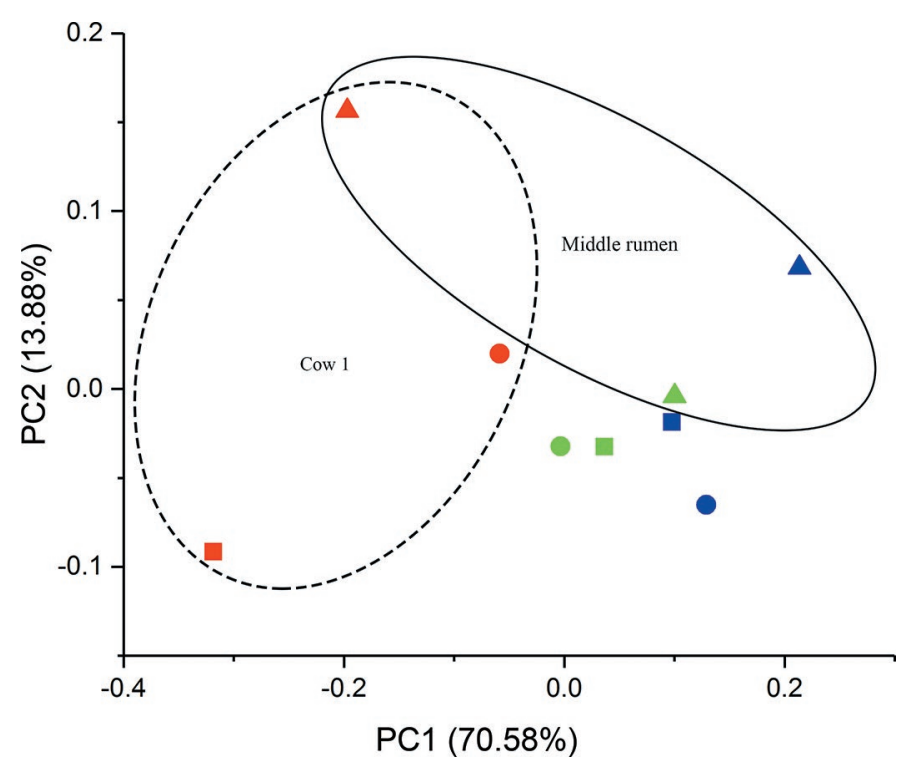

Figure 1. Principal coordinate (PC) analysis plot at operational taxonomic unit level based on Bray-Curtis distances. Cows 1, 2, and 3 were set in a medium, light, and dark scale of gray, respectively. The front, middle, and hind rumen were shown by square (ם), triangle $(\boldsymbol{\Lambda})$, and circle $(\bullet)$, respectively. Dashed circle indicated cow 1 was separated from the other 2 cows, whereas the solid circle indicated that middle rumen was separated from front and hind rumen. Color version available online. 
now called Methanomassiliicoccales according to Borrel et al. (2014). The PCoA results showed ruminal methanogen communities were distinctly different among 3 cows (Figure 1), with relative abundance of Methanobacteriales varying at 57.1 to $90.8 \%$ among 3 cows (Figure 2). Additionally, the PCoA results also showed that methanogen community in the middle rumen tended to be separated from the front and hind rumen (Figure 1). Alpha diversity also indicated that Simpson was lower $(P=0.02$, Table 2$)$ in front rumen compared with middle rumen. The abundance of Methanobacteriales, the dominant order of methanogens, was the highest, whereas the Thermoplasmatales-related order Methanomassiliicoccales was the lowest in the middle rumen among 3 rumen sites (Figure 2). Unfortunately, abundance of Methanobacteriales and Methanomassiliicoccales did not reach statistically significant difference among 3 rumen sites. However, the qPCR results show that Methanobrevibacter, a dominant genus of methanogens in rumen, was the highest $(P=0.03$, Table 2$)$ in front rumen than middle and hind rumen, which consistent with $\mathrm{dCH}_{4}$ concentration and $\mathrm{pH}$ of ruminal fluid among 3 rumen sites.

In summary, contents from the cranial rumen sac had higher $\mathrm{dCH}_{4}$ concentration than middle and hind rumen, which might be caused by greater populations of Methanobrevibacter. The cranial rumen is in close proximity to the esophagus and has higher $\mathrm{pH}$ caused by incoming saliva, and higher $\mathrm{pH}$ likely changed methanogen communities and methanogenesis within the rumen. Variation in fermentation end-products, methanogens populations, and methanogenesis in different rumen sites might need to be considered when colleting rumen samples from nutritional studies.

\section{ACKNOWLEDGMENTS}

This work was supported by the National Natural Science Foundation of China (Grant No. 31561143009, 31320103917 and 31472133; Beijing, China), National

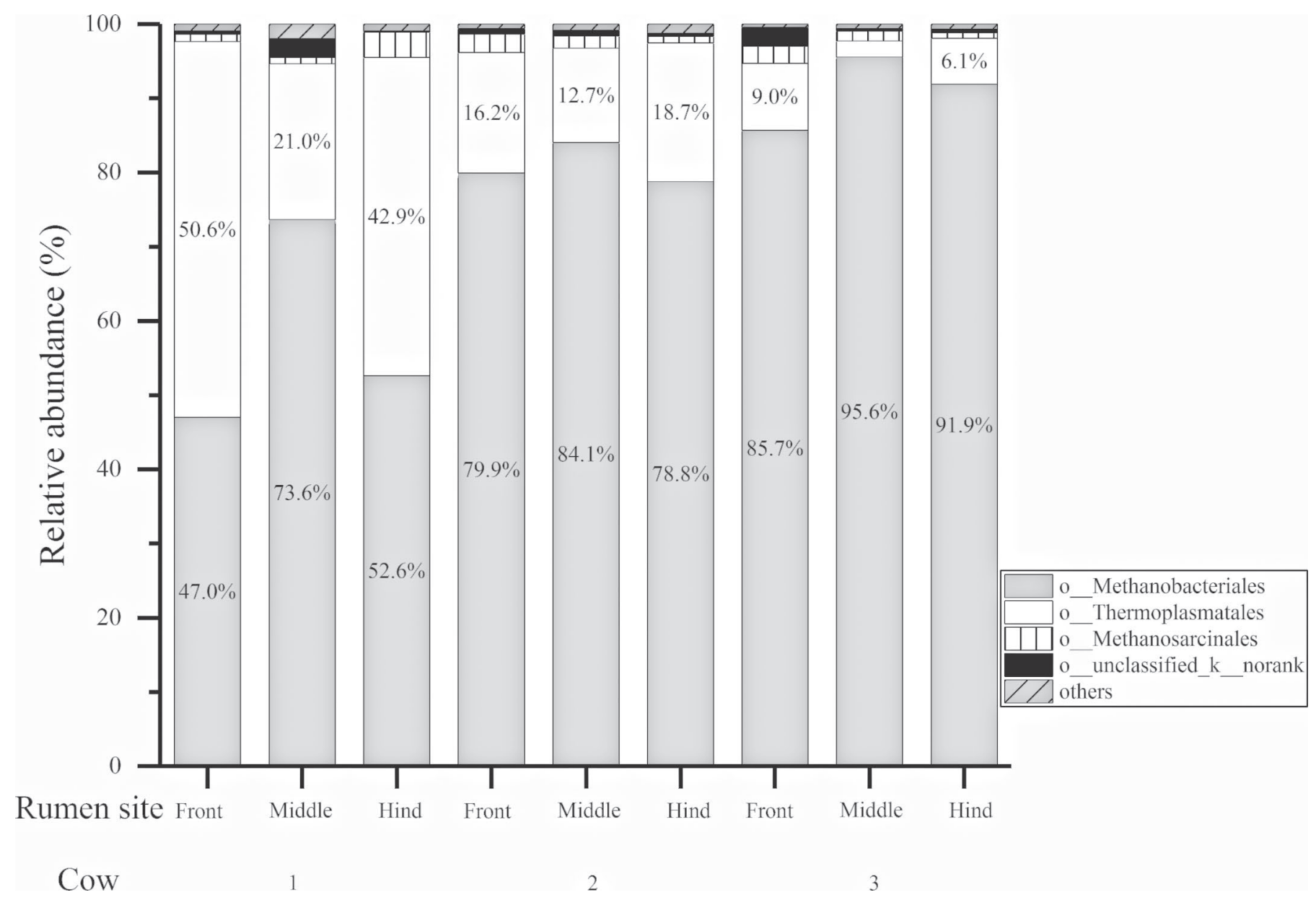

Figure 2. Rumen methanogen community at the order level. The sequences classified to the order Thermoplasmatales against SILVA archaea database v.128 are now called the Methanomassiliicoccales, according to the report of Borrel et al. (2014). 
Key Research and Development Program of China (Grant No. 2016YFD0500504; Beijing, China), State Key Laboratory of Animal Nutrition (2004DA125184F1705; Beijing, China), China Agriculture Research System (Grant No. CARS-36; Beijing, China), Youth Innovation Promotion Association CAS (2016327; Beijing, China), and CAS President's International Fellowship for Visiting Scientists (2017VBA0026; Beijing, China). The authors thank Alexander N. Hristov (The Pennsylvania State University, State College) for his critical review of our manuscript.

\section{REFERENCES}

Aschenbach, J. R., G. B. Penner, F. Stumpff, and G. Gäbel. 2011. Ruminant nutrition symposium: Role of fermentation acid absorption in the regulation of ruminal pH. J. Anim. Sci. 89:1092-1107. https://doi.org/10.2527/jas.2010-3301.

Borrel, G., N. Parisot, H. M. Harris, E. Peyretaillade, N. Gaci, W. Tottey, O. Bardot, K. Raymann, S. Gribaldo, P. Peyret, P. W. O'Toole, and J. F. Brugere. 2014. Comparative genomics highlights the unique biology of Methanomassiliicoccales, a Thermoplasmatales-related seventh order of methanogenic archaea that encodes pyrrolysine. BMC Genomics 15:679. https://doi.org/10 .1186/1471-2164-15-679.

Bray, J. R., and J. T. Curtis. 1957. An ordination of the upland forest communities of southern Wisconsin. Ecol. Monogr. 27:325-349.

Bryant, A. M. 1964. Variations in the $\mathrm{pH}$ and volatile fatty acid concentration within the bovine reticulo-rumen. N. Z. J. Agric. Res. 7:694-706. https://doi.org/10.1080/00288233.1964.10416398.

Caporaso, J. G., K. Bittinger, F. D. Bushman, T. Z. DeSantis, G. L. Andersen, and R. Knight. 2010. PyNAST: A flexible tool for aligning sequences to a template alignment. Bioinformatics 26:266-267. https://doi.org/10.1093/bioinformatics/btp636.

Duffield, T., J. C. Plaizier, A. Fairfield, R. Bagg, G. Vessie, P. Dick, J. Wilson, J. Aramini, and B. McBride. 2004. Comparison of techniques for measurement of rumen $\mathrm{pH}$ in lactating dairy cows. J. Dairy Sci. 87:59-66. https://doi.org/10.3168/jds.S0022 -0302(04)73142-2.

Edgar, R. C. 2013. UPARSE: Highly accurate OTU sequences from microbial amplicon reads. Nat. Methods 10:996-998. https://doi .org/10.1038/nmeth.2604.

Hook, S. E., A. D. Wright, and B. W. McBride. 2010. Methanogens: methane producers of the rumen and mitigation strategies. Archaea 2010:945785. https://doi.org/10.1155/2010/945785.

Huang, X. D., G. Martinez-Fernandez, J. Padmanabha, R. Long, S. E. Denman, and C. S. McSweeney. 2016. Methanogen diversity in indigenous and introduced ruminant species on the Tibetan Plateau. Archaea 2016:5916067. https://doi.org/10.1155/2016/5916067.

Janssen, P. H. 2010. Influence of hydrogen on rumen methane formation and fermentation balances through microbial growth kinetics and fermentation thermodynamics. Anim. Feed Sci. Tech. 160:122. https://doi.org/10.1016/j.anifeedsci.2010.07.002.

Jiao, J., P. Wang, Z. He, S. Tang, C. Zhou, X. Han, M. Wang, D. Wu, J. Kang, and Z. Tan. 2014. In vitro evaluation on neutral detergent fiber and cellulose digestion by post-ruminal microorganisms in goats. J. Sci. Food Agric. 94:1745-1752. https://doi.org/10.1002/ jsfa. 6485.

Kozich, J. J., S. L. Westcott, N. T. Baxter, S. K. Highlander, and P. D. Schloss. 2013. Development of a dual-index sequencing strategy and curation pipeline for analyzing amplicon sequence data on the MiSeq Illumina sequencing platform. Appl. Environ. Microbiol. 79:5112-5120. https://doi.org/10.1128/AEM.01043-13.

Lana, R. P., J. B. Russell, and M. E. Van Amburgh. 1998. The role of $\mathrm{pH}$ in regulating ruminal methane and ammonia production. J. Anim. Sci. 76:2190-2196. https://doi.org/10.2527/1998.7682190x.

Li, M., G. B. Penner, E. Hernandez-Sanabria, M. Oba, and L. L. Guan. 2009. Effects of sampling location and time, and host animal on 
assessment of bacterial diversity and fermentation parameters in the bovine rumen. J. Appl. Microbiol. 107:1924-1934. https://doi .org/10.1111/j.1365-2672.2009.04376.x.

McCracken, T. O., R. A. Kainer, and T. L. Spurgeon. 1999. The ox (Bos taurus, also Bos indicus). Pages 41 in Spurgeon's Color Atlas of Large Animal Anatomy: The Essentials. John Wiley \& Sons, Ames, IA.

Quast, C., E. Pruesse, P. Yilmaz, J. Gerken, T. Schweer, P. Yarza J. Peplies, and F. O. Glockner. 2013. The SILVA ribosomal RNA gene database project: Improved data processing and web-based tools. Nucleic Acids Res. 41:D590-D596. https://doi.org/10.1093/ nar/gks1219.

Rutledge, R. G., and C. Côté. 2003. Mathematics of quantitative kinetic PCR and the application of standard curves. Nucleic Acids Res. 31:e93.

Schloss, P. D., S. L. Westcott, T. Ryabin, J. R. Hall, M. Hartmann, E. B. Hollister, R. A. Lesniewski, B. B. Oakley, D. H. Parks, C. J. Robinson, J. W. Sahl, B. Stres, G. G. Thallinger, D. J. Van Horn, and C. F. Weber. 2009. Introducing mothur: Open-source, platform-independent, community-supported software for describing and comparing microbial communities. Appl. Environ. Microbiol. 75:7537-7541. https://doi.org/10.1128/AEM.01541-09.

Shen, J. S., Z. Chai, L. J. Song, J. X. Liu, and Y. M. Wu. 2012. Insertion depth of oral stomach tubes may affect the fermentation parameters of ruminal fluid collected in dairy cows. J. Dairy Sci. 95:5978-5984. https://doi.org/10.3168/jds.2012-5499.

Wang, M., X. Z. Sun, P. H. Janssen, S. X. Tang, and Z. L. Tan. 2014. Responses of methane production and fermentation pathways to the increased dissolved hydrogen concentration generated by eight substrates in in vitro ruminal cultures. Anim. Feed Sci. Technol. 194:1-11. https://doi.org/10.1016/j.anifeedsci.2014.04.012.
Wang, M., R. Wang, P. H. Janssen, X. M. Zhang, X. Z. Sun, D. Pacheco, and Z. L. Tan. 2016a. Sampling procedure for the measurement of dissolved hydrogen and volatile fatty acids in the rumen of dairy cows. J. Anim. Sci. 94:1159-1169. https://doi.org/10.2527/ jas.2015-9658.

Wang, M., R. Wang, T. Xie, P. H. Janssen, X. Sun, K. A. Beauchemin, Z. Tan, and M. Gao. 2016b. Shifts in rumen fermentation and microbiota are associated with dissolved ruminal hydrogen concentrations in lactating dairy cows fed different types of carbohydrates. J. Nutr. 146:1714-1721. https://doi.org/10.3945/jn.116 .232462 .

Wang, Q., G. M. Garrity, J. M. Tiedje, and J. R. Cole. 2007. Naive Bayesian classifier for rapid assignment of rRNA sequences into the new bacterial taxonomy. Appl. Environ. Microbiol. 73:52615267. https://doi.org/10.1128/AEM.00062-07.

Weatherburn, M. W. 1967. Phenol-hypochlorite reaction for determination of ammonia. Anal. Chem. 39:971-974. https://doi.org/10 $.1021 /$ ac60252a045.

Wenner, B. A., J. de Souza, F. Batistel, T. J. Hackmann, Z. Yu, and J. L. Firkins. 2017. Association of aqueous hydrogen concentration with methane production in continuous cultures modulated to vary $\mathrm{pH}$ and solids passage rate. J. Dairy Sci. 100:5378-5389. https://doi.org/10.3168/jds.2016-12332.

Yu, Y., C. Lee, J. Kim, and S. Hwang. 2005. Group-specific primer and probe sets to detect methanogenic communities using quantitative real-time polymerase chain reaction. Biotechnol. Bioeng. 89:670-679. https://doi.org/10.1002/bit.20347.

Yu, Z., and M. Morrison. 2004. Improved extraction of PCR-quality community DNA from digesta and fecal samples. Biotechniques $36: 808$. 